Check for updates

Cite this: RSC Adv., 2017, 7, 54995

Received 19th September 2017 Accepted 25th November 2017

DOI: $10.1039 / c 7 r a 10394 h$

rsc.li/rsc-advances

\section{An amorphous dual action electrocatalyst based on oxygen doped cobalt sulfide for the hydrogen and oxygen evolution reactions $\uparrow$}

\begin{abstract}
Ummul K. Sultana, Tianwei He, Aijun Du (D) and Anthony P. O'Mullane (D)*
The fabrication of electrocatalysts that are active for more than one of the water splitting reactions has gained significant momentum. Here we demonstrate such a material produced via an electrochemical process that is based on amorphous cobalt sulfide films doped with oxygen which are active for the hydrogen evolution reaction (HER) and oxygen evolution reaction (OER) under alkaline conditions. The optimum electrochemical protocol was found to be a repetitive potential cycling approach rather than a constant potential to create an amorphous $\mathrm{CoS}_{x}$ film containing oxygen. Samples with a Co : S ratio of 1.56: 1 were found to be active for the HER in $0.5 \mathrm{M} \mathrm{H}_{2} \mathrm{SO}_{4}$, phosphate buffer and $0.1 \mathrm{M} \mathrm{NaOH}$. Significantly this activity is comparable to highly crystalline nanomaterials of cobalt sulfide. Density functional theory calculations indicated that a reduced S-Co coordination number, as encountered in amorphous materials, leads to an optimum binding energy for hydrogen adsorption on the material which facilitates good electron transfer kinetics. In addition, this material was also active for the OER in alkaline conditions with evidence of conversion to cobalt oxide which gave a low overpotential of $370 \mathrm{mV}$ for an applied current density of $10 \mathrm{~mA} \mathrm{~cm}{ }^{-2}$ with a Tafel slope of $67 \mathrm{mV} \mathrm{dec}^{-1}$. This simple approach shows promise for the fabrication of a dual action electrocatalyst for electrochemical water splitting under alkaline conditions.
\end{abstract}

\section{Introduction}

The ever increasing global demand for energy can only be satisfied in an environmentally sustainable manner with the rapid uptake of clean renewable energy sources. It is well documented that the storage of intermittent energy from sources such as wind and solar is urgently required. Fortunately, there are several approaches which can be undertaken to tackle this issue. One such approach that has been identified since the 1970s is using non-polluting hydrogen as the main energy carrier, where the overall system was termed as the hydrogen economy by Bockris. ${ }^{1}$ Although there are many ways to produce hydrogen, doing so in an environmentally friendly manner is paramount for realising such an economy. Therefore, the generation of hydrogen via electrochemically splitting water into hydrogen and oxygen has received significant attention because the electrolyser can be powered by electricity generated from renewable energy sources. During electrolysis the hydrogen evolution reaction (HER) can only occur in an efficient manner when a suitable electrocatalyst is used. ${ }^{2}$ It is well

School of Chemistry, Physics and Mechanical Engineering, Queensland University of Technology (QUT), GPO Box 2434, Brisbane, QLD 4001, Australia. E-mail: anthony. omullane@qut.edu.au

$\dagger$ Electronic supplementary information (ESI) available. See DOI: 10.1039/c7ra10394h established that the platinum group metals including $\mathrm{Pt}, \mathrm{Rh}$ and Ir are the most active electrocatalysts for this reaction. ${ }^{3}$ Although alternative catalysts to these precious metals can achieve high efficiency and activity, sustaining this for prolonged periods is still quite a challenge, ${ }^{4-13}$ and therefore has attracted much attention. ${ }^{14-31}$ There are quite a number of low cost and earth abundant materials that have been investigated, including transition metal alloys, ${ }^{32-34}$ transition metal oxides and hydroxides, ${ }^{35-39}$ chalcogenides, ${ }^{40,41}$ carbides, ${ }^{42-49}$ and phosphides $^{50-52}$ as well as entirely non-metallic catalysts. ${ }^{53,54}$

In particular, cobalt based compounds such as sulfides, ${ }^{55,56}$ nanocomposites of $\operatorname{CoS}_{2}$ using reduced graphene oxide (RGO) and carbon nanotubes (CNT), ${ }^{37}$ amorphous $\mathrm{CoS}_{x}$ composed of oxide and sulphide clusters, ${ }^{57}$ phosphides, ${ }^{49,58-60}$ phosphosulphides, ${ }^{61}$ and selenide complexes like $\operatorname{CoS}_{2 x} \mathrm{Se}_{2(1-x)}$ (ref. 62) have been identified as robust electrocatalysts for the HER. For synthesizing these HER catalysts most researchers have adopted wet chemical methods, vapour deposition, ${ }^{63}$ annealing ${ }^{64}$ or hydrothermal synthesis followed by temperature programmed reduction (TPR) ${ }^{65}$ However, electrochemically deposited nanomaterials have been shown to also perform as effective HER catalysts. The advantage of this approach is that it easy to perform, scalable, does not require expensive equipment and can be undertaken under ambient conditions. ${ }^{66}$ It also has a number of freedom parameters to tune the composition, morphology and density of active sites which is another major 
advantage. ${ }^{67-73}$ Recent theoretical work by Kornienko et al. suggested that amorphous $\operatorname{CoS}_{x}$ where oxygen is incorporated into the material is also a candidate for the HER ${ }^{57}$ Shanmugam et al. have reported that electrodeposited nickel iron sulphides on nickel foam ${ }^{74}$ and hydrothermally grown $\mathrm{NiCo}_{2} \mathrm{~S}_{4}$ nanowires on nickel foam ${ }^{75}$ show bifunctional activity for the HER and the OER.

In addition there has been significant interest in developing materials that are not only active for the HER but also the oxygen evolution reaction (OER). In this area the majority of cobalt based materials reported are the oxides, hydroxides or oxy-hydroxides rather than sulphide based moieties. Cai et al. have demonstrated that oxygen incorporated into cobalt sulphide nanocubes are active for the OER, although the method of synthesis was quite involved using several chemical steps and high temperature annealing. ${ }^{76}$ However this material was not tested for the HER and to date no cobalt sulfide based materials have been reported that are active for both HER and OER. In this work we address this and use a simple and rapid electrochemical approach to deposit amorphous cobalt sulphide materials that contain oxygen, which are active for both HER and OER under alkaline conditions.

\section{Experimental}

\section{Chemicals}

Cobalt(II) nitrate hydrated (Chem-Supply) and thiourea (SigmaAldrich) were used as received without any further purification. Sulfuric acid (96\%) was purchased from EMD chemicals. For each experiment and other washing purposes Milli-Q water (resistivity of 18.2 M $\Omega \mathrm{cm}$ ) from a Millipore Milli-Q system was used. Analytical grade chemicals and Milli-Q water were used for preparing all electrolyte solutions.

\section{Preparation of the cobalt sulphide film on glassy carbon and ITO}

All the experiments were conducted using a conventional three electrode system. While depositing on glassy carbon, the working electrode was glassy carbon (area was $0.071 \mathrm{~cm}^{2}$, Bioanalytical Systems, Inc.), platinum (Bioanalytical Systems, Inc.) was the counter electrode and $\mathrm{Ag} \mid \mathrm{AgCl}(3 \mathrm{M} \mathrm{KCl}$, Bioanalytical Systems, Inc.) was the reference electrode. Some electrodeposition experiments were also performed on indium doped tin oxide (ITO) films (Delta Technologies 4-10 $\Omega \mathrm{sq}^{-1}$ ) and were cut into $1 \mathrm{~cm} \times 1 \mathrm{~cm}$ pieces. All the cyclic voltammetry and chronoamperometry experiments were conducted using a BioLogic VSP workstation operated by EC-lab software (version 10.44). Before every experiment the exposed area of the GC working electrode was wet polished with $0.3 \mu \mathrm{m}$-sized alumina powder on a micro cloth and was rinsed with Milli-Q water. Before performing any electrochemical experiment the solutions were purged with nitrogen gas. Different $\mathrm{pH}$ levels of the electrolyte solution were adjusted and then measured by a Metrohm 826 pH meter. The electrodeposition experiments were carried out by cyclic voltammetry and chronoamperometry techniques. After each deposition the films were washed with Milli-Q water and dried with pure nitrogen gas to remove any electrolyte solution from the surface. For all the electrochemical data the potential was converted to the RHE scale via the following formula, $E_{\mathrm{RHE}}=E_{\mathrm{Ag} / \mathrm{AgCl}}+0.059 \times \mathrm{pH}+0.197 \mathrm{~V}$. The current density reported in this work was normalized to the geometric surface area of the underlying electrode.

\section{Surface characterisation}

X-ray photoelectron spectroscopy data were collected using an Omicron Multiscan Lab Ultra-high vacuum scanning tunnelling microscope (UHV-STM) where a $125 \mathrm{~mm}$ hemispherical electron energy analyser was incorporated. Non-monochromatic $\mathrm{Mg}$ $\mathrm{K} \alpha(1253.6 \mathrm{eV}) \mathrm{X}$-ray source (DAR 400, Omicron Nanotechnology) was used for XPS experiments and the incident angle was $65^{\circ}$ to the surface of the sample. The analyser passed energy of $50 \mathrm{eV}$ with steps of $0.5 \mathrm{eV}$ and the dwell time was $200 \mathrm{~ms}$. High-resolution scans with a narrow region for Co 2p, C 1s and $\mathrm{O} 1 \mathrm{~s}$ were taken at $20 \mathrm{eV}$ pass energy, $0.1 \mathrm{eV}$ steps and with a 200 ms dwell time. Besides a wide scan of low binding energy region was performed from $250 \mathrm{eV}$ to $0 \mathrm{eV}$ swept at high resolution. The base pressure in the analysis chamber was $1.0 \times 10^{-9}$ Torr. But the pressure was $1.0 \times 10^{-8}$ Torr while the measurement was done. Atomic compositions of the surface were calculated using the CasaXPS version 2.3.15 software and a linear baseline with Kratos library Relative Sensitivity Factors (RSFs). EDX was performed on a FEI Quanta 200 Environmental SEM at an operating voltage of $20 \mathrm{kV}$. Around $100 \mathrm{~nm}$ thick samples were prepared by the electrodeposition method. X-ray diffraction (XRD) patterns of the sample were collected using a Philips PANalytical X'pert Pro diffractometer. CuK $\alpha$ radiation $(\lambda=$ $1.5418 \AA$ A) and a fixed power source ( $40 \mathrm{kV}$ and $40 \mathrm{~mA})$ were used. Images were captured using a Helium Ion Microscope (HIM) ORION NanoFab while the chamber pressure was $2 \times 10^{-7}$ Torr, the gun pressure of gas field ion source (GFISGun) was $5 \times$ $10^{-10}$ Torr and the column pressure (GFISColumn) was $9 \times$ $10^{-9}$ Torr.

\section{Computational details}

Density functional theory (DFT) as implemented in the Vienna $A b$ initio Simulation Package (VASP) code were employed to perform the calculations. ${ }^{77,78} \mathrm{We}$ used the generalized gradient approximation ${ }^{79}$ in the form of the Perdew-Burke-Ernzerhof functional ${ }^{80}$ to describe exchange-correlation interactions. Blöchl's all-electron, frozen-core projector augmented wave (PAW) method ${ }^{\mathbf{8 1}}$ was used to represent nuclei and core electrons. In all calculations, the van der Waals interaction was described by using the empirical correction in Grimme's scheme, i.e., DFT + D3 ${ }^{82}$ The electron wave functions were expanded using the plane waves with a cut off energy of $500 \mathrm{eV}$. The geometries were optimized until the energy and the force were converged to $0.001 \mathrm{eV} \AA^{-1}$ and $10^{-6} \mathrm{eV}$, respectively. For the $k$-point sampling, we used a single $\Gamma$ point mesh $1 \times 1 \times 1$ in reciprocal space during geometry optimization. 


\section{The calculation of hydrogen binding free energy}

The free energy of the adsorption atomic hydrogen $\left(\Delta G_{\mathrm{H}^{*}}^{0}\right)$ is calculated as:

$$
\Delta G_{\mathrm{H}^{*}}^{0}=\Delta E_{\mathrm{H}}+\Delta E_{\mathrm{ZPE}}-T \Delta S_{\mathrm{H}}
$$

$\Delta E_{\mathrm{H}}$ represents the differential hydrogen adsorption energy and can be described by:

$$
\Delta E_{\mathrm{H}}=E_{\mathrm{H}^{*}}-E_{*}-1 / 2 E_{\mathrm{H}_{2}}
$$

where $*$ denotes the catalyst. $E_{\mathrm{H}^{*}}, E_{*}$ and $E_{\mathrm{H}_{2}}$ represents total energies of catalyst plus one $\mathrm{H}$ adsorbed hydrogen atoms, the total energies of catalyst without adsorbed hydrogen atoms and $\mathrm{H}_{2}$ gas, respectively. $\Delta E_{\mathrm{ZPE}}$ is the difference corresponding to the zero point energy between the adsorbed state and the gas phase. The contributions from the catalysts to both $\Delta E_{\mathrm{ZPE}}$ and $\Delta S_{\mathrm{H}}$ are small and can be neglected. Therefore, $\Delta E_{\mathrm{ZPE}}$ is obtained by: ${ }^{83}$

$$
\Delta E_{\mathrm{ZPE}}=E_{\mathrm{ZPE}}^{\mathrm{H}}-1 / 2 E_{\mathrm{ZPE}}^{\mathrm{H}_{2}}
$$

where, $E_{\mathrm{ZPE}}^{\mathrm{H}}$ is the zero-point energy of one adsorbed atomic hydrogens on the catalyst without the contribution of the catalyst. $E_{\mathrm{ZPE}}^{\mathrm{H}_{2}}$ is the zero-point energy of $\mathrm{H}_{2}$ in the gas phase.

$S_{\mathrm{H}_{2}}^{0}$ is the entropy of $\mathrm{H}_{2}$ gas at the standard condition. ${ }^{84}$ The $\Delta S_{\mathrm{H}}$ can be obtained by:

$$
\Delta S_{\mathrm{H}} \cong-1 / 2 S_{\mathrm{H}_{2}}^{0}
$$

The calculated vibrational frequency for $\mathrm{H}_{2}$ gas is $4390 \mathrm{~cm}^{-1}$, the vibrational frequency of $\mathrm{H}$ adsorbed on $\mathrm{CoS}_{2}$ are $2523 \mathrm{~cm}^{-1}$, $662 \mathrm{~cm}^{-1}$, and $381 \mathrm{~cm}^{-1}$. Therefore the overall corrections are taken as:

$$
\Delta G_{\mathrm{H}^{*}}^{0}=\Delta E_{\mathrm{H}}+0.25 \mathrm{eV}
$$

\section{Results and discussion}

The electrodeposition of cobalt species from $\mathrm{Co}\left(\mathrm{NO}_{3}\right)_{2}$ in the absence and presence of thiourea at different solution $\mathrm{pH}$ is shown in Fig. 1. In the absence of thiourea a large cathodic process is observed on the forward sweep at all $\mathrm{pH}$ values which is followed by current crossover in the reverse sweep. This is highly indicative of a nucleation growth process occurring and a deposit being formed on the electrode surface.$^{85}$ The reactions involved can be outlined by the following equations: ${ }^{86,87}$

$$
\begin{gathered}
2 \mathrm{H}_{2} \mathrm{O}+2 \mathrm{e}^{-} \rightarrow \mathrm{H}_{2}+2 \mathrm{OH}^{-} \\
2 \mathrm{Co}_{(\mathrm{aq})}^{2+}+2 \mathrm{e}^{-} \rightarrow \mathrm{Co}(\mathrm{OH})_{2(\mathrm{ads})} \\
\mathrm{Co}(\mathrm{OH})_{2(\mathrm{ads})}+2 \mathrm{e}^{-} \rightarrow \mathrm{Co}_{(\mathrm{s})}+2 \mathrm{OH}^{-}
\end{gathered}
$$

A small anodic process is observed on the reverse sweep which can be attributed to the reverse of eqn (7). The position of this peak varies slightly with the $\mathrm{pH}$ of the electrolyte. In the presence of thiourea (TU) a significant change occurs in the
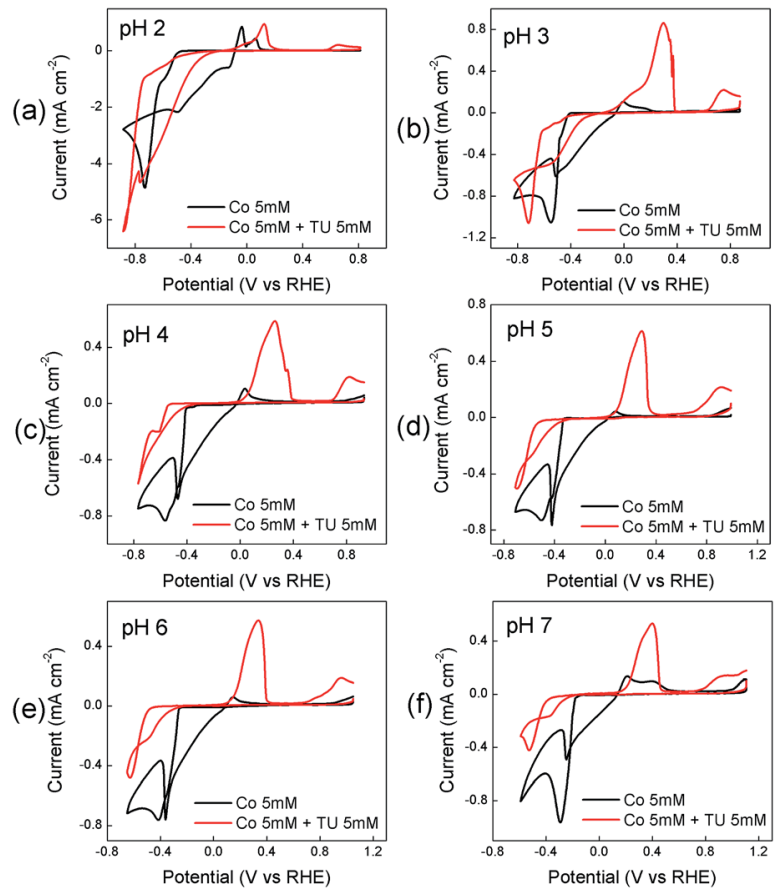

Fig. 1 Effect of TU on the cyclic voltammetric response recorded at a GC electrode in solutions containing $0.005 \mathrm{M} \mathrm{Co(॥)} \mathrm{only} \mathrm{and} 0.005 \mathrm{M}$ $\mathrm{Co}($ II) $+0.005 \mathrm{M}$ thiourea at different $\mathrm{pH}$ values.

cyclic voltammetric response. The magnitude of the cathodic process decreases at all $\mathrm{pH}$ values and is shifted to more negative potentials. This can be attributed to the formation of $\mathrm{CoS}_{x}$ via the following processes: ${ }^{88}$

$$
\begin{gathered}
\mathrm{CS}\left(\mathrm{NH}_{2}\right)_{2}+2 \mathrm{OH}^{-} \rightarrow \mathrm{S}^{2-}+\mathrm{OC}\left(\mathrm{NH}_{2}\right)_{2}+\mathrm{H}_{2} \mathrm{O} \\
\mathrm{Co}^{2+}+\mathrm{S}^{2-} \rightarrow \mathrm{CoS}_{x(\mathrm{ads})}
\end{gathered}
$$

The electrochemically generated hydroxide ions from the reduction of water have been reported to react with thiourea to liberate sulphide ions which complex with $\mathrm{Co}^{2+}$ ions in solution to form $\operatorname{CoS}_{x}$ which precipitates onto the electrode surface. However a recent study has concluded that in fact metal sulphides are synthesised via the formation of a $\left(\mathrm{NH}_{2}\right)_{2} \mathrm{CS}_{\mathrm{s}}-$ $\mathrm{M}^{2+}-\mathrm{OH}_{\mathrm{h}}{ }^{-}$complex which decomposes into the relevant metal sulphide via the following process: ${ }^{89}$

$$
\begin{array}{r}
\mathrm{M}^{2+}+\mathrm{CS}\left(\mathrm{NH}_{2}\right)_{2}+2 \mathrm{OH}^{-} \rightleftarrows \underset{\mathrm{M}(\mathrm{OH})_{2} \mathrm{CS}\left(\mathrm{NH}_{2}\right)_{2} \rightarrow}{\mathrm{MS}+\mathrm{H}_{2} \mathrm{NCN}+2 \mathrm{H}_{2} \mathrm{O}}
\end{array}
$$

On the reverse sweep a prominent oxidation peak can be seen in the presence of TU and is attributed to the oxidation of thiourea to formamide disulphide via: ${ }^{88}$

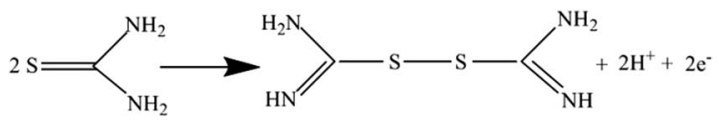

After this process a smaller peak is observed towards the end of the sweep at all $\mathrm{pH}$ values and is attributed to the oxidation of 
$\mathrm{CoS}_{x}$ on the electrode surface ${ }^{\mathbf{9 0}}$ (this process is absent when TU is not present in the solution). Overall the behaviour over a $\mathrm{pH}$ range from 3-7 is quite similar showing distinct processes for TU oxidation to FD and the oxidation of $\operatorname{CoS}_{x}$ at higher potential values. At $\mathrm{pH} 2$ both of these processes are suppressed and indicates that thiourea is less susceptible to oxidation and also that the formation of $\mathrm{CoS}_{x}$ is more inhibited.

It is well established that the potential waveform used to electrodeposit materials has a significant impact on morphology and electrocatalytic activity. Therefore chronoamperometry and repetitive potential cycling were chosen as methods to electrodeposit $\mathrm{CoS}_{x}$ films. Fig. S1† shows currenttime transients for the electrodeposition of $\mathrm{CoS}_{x}$ at different $\mathrm{pH}$ values and Fig. $\mathrm{S} 2 \uparrow$ shows the subsequent anodic stripping voltammograms for the deposit on the electrode surface. This is consistent with the data shown in Fig. 1 where a significant oxidation process for $\operatorname{CoS}_{x}$ oxidation occurred from $0.70 \mathrm{~V}$ over a $\mathrm{pH}$ range of 4-6. If the TU concentration was increased up to $0.5 \mathrm{M}$ then the amount of $\operatorname{CoS}_{x}$ formation increased, as evidence by the increased oxidation process at $c a .0 .80 \mathrm{~V}$ at all $\mathrm{pH}$ values (Fig. S3 $\dagger$ ). The presence of $\operatorname{CoS}_{x}$ was confirmed by EDS and XPS analysis as discussed later. It was also found that at $\mathrm{pH}$ values of 6-7 a precipitate of $\mathrm{Co}(\mathrm{OH})_{2}$ formed slowly in solution and therefore was not investigated further in electrodeposition reactions. Repetitive potential cycling has been used in many studies to create highly textured surfaces which have increased surface area and electrocatalytic activity for a variety of reactions. This was shown to be good method to synthesise nickel iron sulphides which were active for both HER and OER. ${ }^{74}$ Therefore a similar approach was undertaken here and shown in Fig. 2a are repetitive cyclic voltammograms recorded at an ITO electrode in the presence of $5 \mathrm{mM} \mathrm{Co}^{2+}$ and $5 \mathrm{mM}$ TU where the $\mathrm{pH}$ was not adjusted and measured to be 5.6. The anodic potential limit was decreased to avoid any $\operatorname{CoS}_{x}$ oxidation and allow the build-up of the deposit on the electrode surface. The cathodic potential limit was also curtailed to avoid the formation of any cobalt metal on the electrode surface. On the first cycle (initiated at positive potential) the same behaviour as seen in Fig. 1 (pH 5 or 6) is observed whereby a cathodic process is observed at ca. $-0.40 \mathrm{~V}\left(\mathrm{C}_{2}\right)$ due to the formation of $\operatorname{CoS}_{x}$. On the anodic scan a large peak $\mathrm{A}_{1}$ is observed due to the oxidation of TU to FD. Upon cycling and taking the $3^{\text {rd }}$ cycle as an example a new peak $\mathrm{C}_{1}$ appears which is due to the reduction of FD back
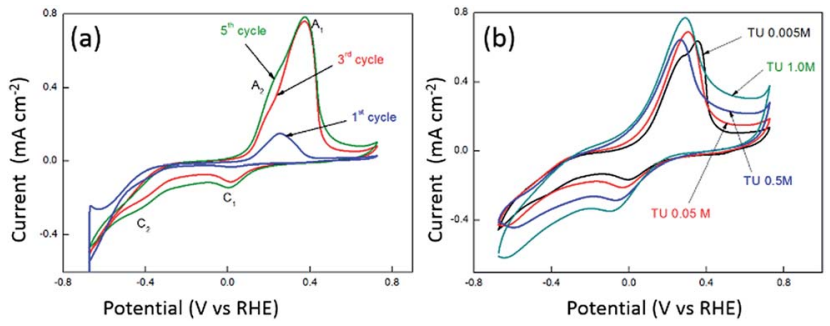

Fig. 2 Cyclic voltammetry recorded at a glassy carbon performed from -0.67 to $0.72 v s$. RHE at a scan rate of $15 \mathrm{mV} \mathrm{s}^{-1}$ in a solution

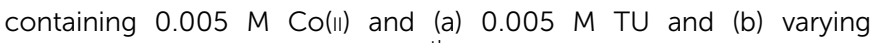
concentration of $\mathrm{TU}$ where the $15^{\text {th }}$ cycle is shown. to $\mathrm{TU}$ (note its absence of the $1^{\text {st }}$ cycle as TU was not oxidised). The magnitude of process $\mathrm{C}_{2}$ also increases in the $3^{\text {rd }}$ cycle illustrating the growth of the $\operatorname{CoS}_{x}$ film. This growth in process $\mathrm{C}_{2}$ can also be observed in the $5^{\text {th }}$ cycle. The effect of TU concentration is shown in Fig. $2 \mathrm{~b}$ where the $15^{\text {th }}$ cycle is shown. It can be seen that the magnitude of the $\mathrm{C}_{2}$ process increases with TU concentration indicating the formation of a greater amount of $\operatorname{CoS}_{x}$ on the electrode surface.

The morphology, composition and their effect on electrocatalytic performance for the HER was investigated for samples electrodeposited on a GC electrode using chronoamperometry (10 min deposition time) and repetitive cycling (15 cycles at $15 \mathrm{mV} \mathrm{s}^{-1}$, Fig. $\mathrm{S} 4 \dagger$ ) at $\mathrm{pH}$ values of 3 and 5.6 at a constant $\mathrm{Co}^{2+}$ concentration of $5 \mathrm{mM}$ and $\mathrm{TU}$ ranging from $5 \mathrm{mM}$ to $1 \mathrm{M}$. With the CA technique the surface morphologies are quite different with the change of solution $\mathrm{pH}$. At $\mathrm{pH} 3$ for all TU concentrations the structures are more globular like (Fig. 3a1-d1) whereas
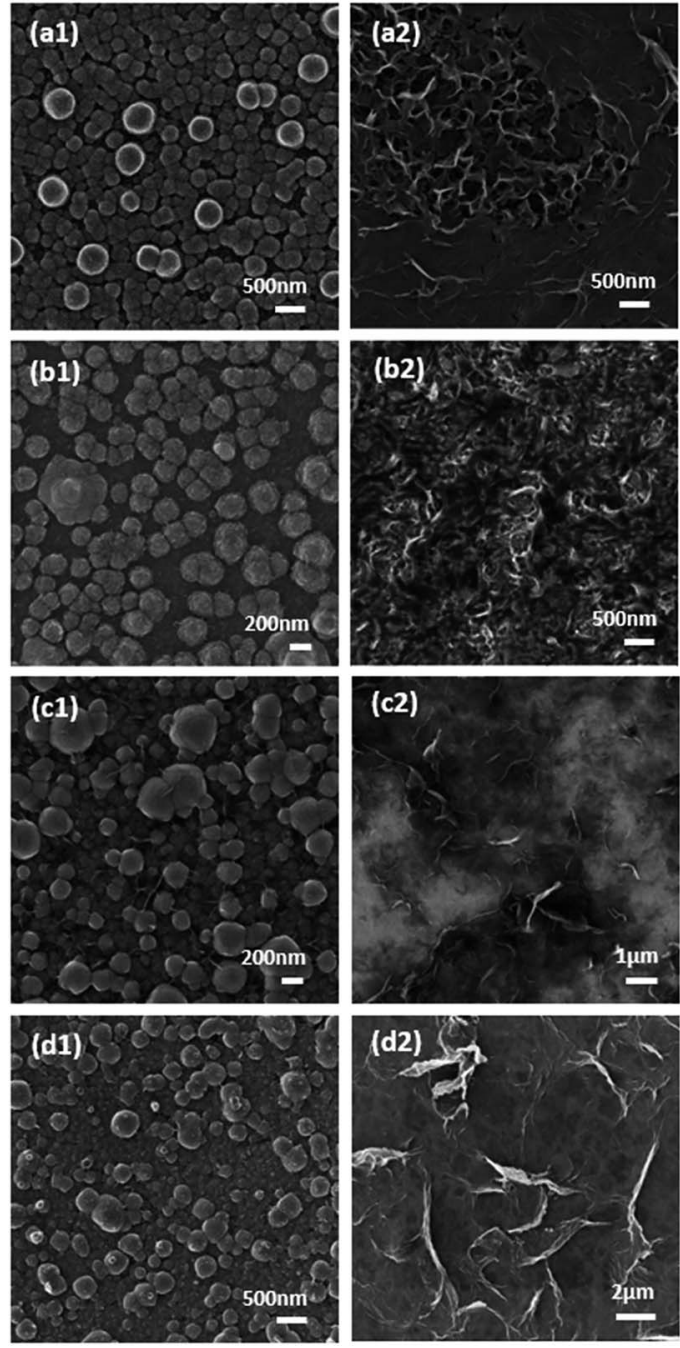

Fig. 3 Helium Ion Microscope (HIM) images of $\operatorname{CoS}_{x}$ films deposited on glassy carbon via chronoamperometry in a solution containing $0.005 \mathrm{M}$ of $\mathrm{Co}(\mathrm{II})$ and (a) $0.005 \mathrm{M}$ of thiourea, (b) $0.05 \mathrm{M}$ of thiourea (c) $0.5 \mathrm{M}$ of thiourea and (d) $1.0 \mathrm{M}$ of thiourea at $\mathrm{pH} 3$ (a1-d1) at a constant potential of $-0.72 \mathrm{~V}$ for 10 minutes and at $\mathrm{pH} 5.6(\mathrm{a} 2-\mathrm{d} 2)$ at a constant potential of -0.57 for 10 minutes, respectively. 
they have a distinct layered surface when the electroplating solution $\mathrm{pH}$ is 5.6 (Fig. 3a2-d2) which is highly indicative of the formation of cobalt sulfide. ${ }^{91}$ The layered structure is observed for all concentrations of TU that were used, however the density of the film increases with TU concentration.

When a repetitive cycling protocol was used it can be seen that the formation of layered materials is favoured under nearly all conditions (Fig. 4). The only exception is when a low TU concentration of $5 \mathrm{mM}$ is used at $\mathrm{pH} 3$ where more globule like structures are seen like in the case of chronoamperometric deposition (Fig. 4a1). When the concentration of TU was increased to $0.05 \mathrm{M}$ (Fig. 4b1) small layered deposits can be seen surrounding the main globule like deposits. At the higher TU concentrations the whole surface is dominated by layered structures. At pH 5.6 there is no evidence of globule like deposits and the thickness of the individual flake like deposits increases substantially with TU concentration until a thick and
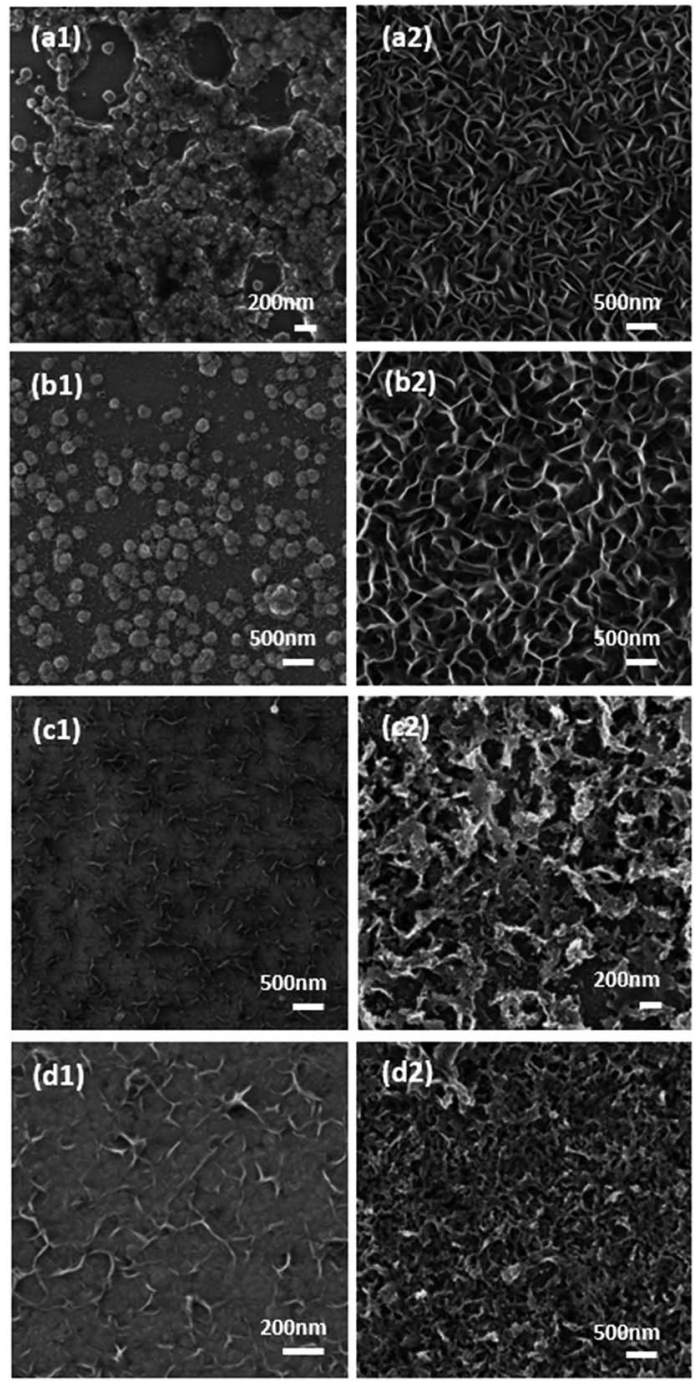

Fig. 4 Helium Ion Microscope (HIM) images of $\operatorname{CoS}_{x}$ films deposited on a glassy carbon electrode via cyclic voltammetry at a scan rate of $15 \mathrm{mV} \mathrm{s}^{-1}$ for 15 cycles in a solution containing $0.005 \mathrm{M}$ of $\mathrm{Co}(॥)$ and (a) $0.005 \mathrm{M}$ of thiourea, (b) $0.05 \mathrm{M}$ of thiourea (c) $0.5 \mathrm{M}$ of thiourea and (d) $1.0 \mathrm{M}$ of thiourea at $\mathrm{pH} 3(\mathrm{a} 1-\mathrm{d} 1)$ and at $\mathrm{pH} 5.6(\mathrm{a} 2-\mathrm{d} 2)$ respectively. densely packed film is formed when $1 \mathrm{M}$ TU was used (Fig. $4 \mathrm{~d} 2$ ). Interestingly, it was found that all the samples synthesized through either chronoamperometry or repetitive cycling were XRD amorphous. A representative XRD pattern for the sample shown in Fig. 4c2 is presented in Fig. S5† where no diffraction peaks were observed. The presence of $\mathrm{Co}, \mathrm{S}$ and $\mathrm{O}$ was confirmed by EDX analysis.

The freshly electrodeposited $\operatorname{CoS}_{x}$ films were initially investigated as HER catalysts in acidic solution to identify the best material. Fig. 5a and b shows linear polarization curves recorded at the samples discussed in Fig. 3 and 4. It is immediately clear that the samples produced via constant potential deposition are not particularly active for the HER. From Fig. 5, it is evident that the samples prepared by the cyclic voltammetric protocol were much more active than those prepared by chronoamperometry and can be attributed to the significantly different deposit created on the electrode surface. The formation of this layered type material is highly critical to good HER performance. The best sample was prepared using $0.5 \mathrm{M}$ TU at a pH of 5.6 (Fig. 5d). The onset potential is $-0.22 \mathrm{~V}$ which is comparable to previous work reported for hollow cobalt sulphide nanomaterials. ${ }^{92}$ However a lower Tafel slope of $72 \mathrm{mV} \mathrm{dec}^{-1}$ was determined for our catalyst compared to $97 \mathrm{mV} \mathrm{dec}{ }^{-1}$ in the aforementioned work. This suggests a Volmer-Tafel mechanism where the Volmer step is the rate limiting step. ${ }^{93}$ However, Tafel slopes in this case are only used as a guide to indicate the possible rate determining step. Care must be taken in

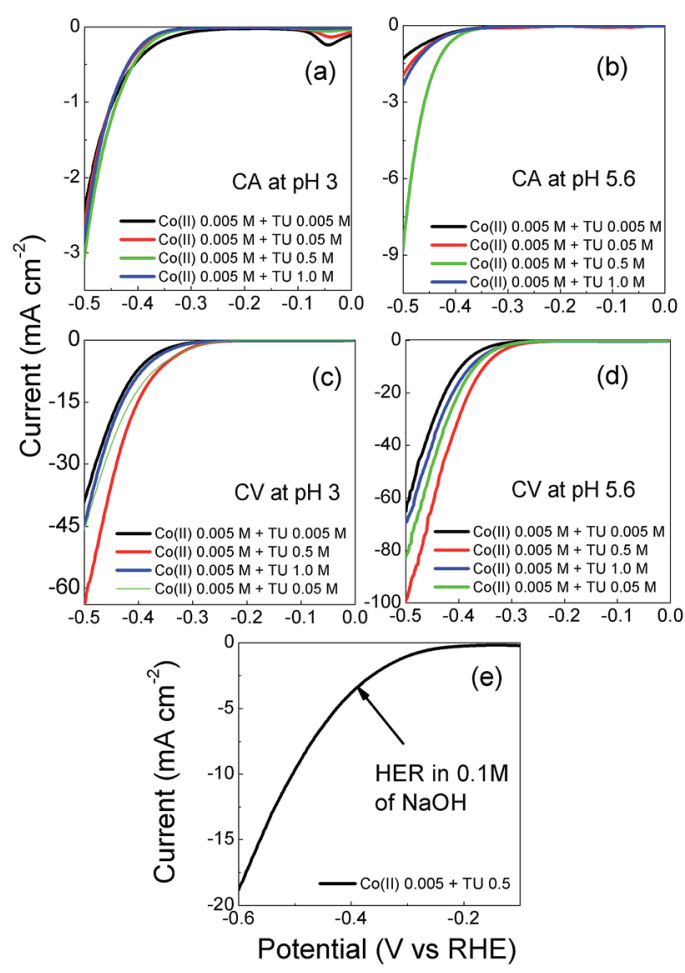

Fig. 5 Linear sweep voltammograms recorded at a GC electrode

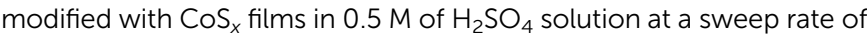
$5 \mathrm{mV} \mathrm{s}^{-1}$ synthesized through chronoamperometry at (a) $\mathrm{pH} 3$ and (b) pH 5.6 and cyclic voltammetry at (c) pH 3 and (d) $\mathrm{pH}$ 5.6; (e) HER for $\mathrm{CoS}_{x}$ performed in $0.1 \mathrm{M} \mathrm{NaOH}$. 
interpreting such values as calculations are based on strict assumptions that do not always hold, hence the wide variation in Tafel slopes that are quoted for metal sulphide materials. The closest Tafel slope reported for cobalt sulphide materials that matches our work is by Sun et al. who reported a value of $72 \mathrm{mV} \mathrm{dec}{ }^{-1}$ for highly crystalline cubic cattierite $\mathrm{CoS}_{2}$ produced via a hydrothermal method. ${ }^{94}$ It is therefore interesting to note that an amorphous material can demonstrate comparable behaviour to well defined crystalline materials for the HER. We also investigated this sample for the HER in alkaline solution (Fig. 5e) where it was found that it also maintained activity under these conditions. A Tafel slope of $142 \mathrm{mV} \mathrm{\textrm {dec } ^ { - 1 }}$ was determined which is also consistent with crystalline materials. ${ }^{94}$ In addition this material also performed well at neutral $\mathrm{pH}$ conditions and showed long term stability over a $24 \mathrm{~h}$ period (Fig. S6 $\dagger$ ) which is a significant improvement over previous work where amorphous $\operatorname{CoS}_{x}$ films were unstable in neutral conditions and required annealing and electrochemical polarisation to ensure stability. ${ }^{56}$

To gain further insights as to why the samples produced by repetitive cycling are more active than those formed under constant potential conditions an XPS study was conducted. Illustrated in Fig. 6a are the Co 2p spectra for samples prepared with different amounts of $\mathrm{TU}$ in solution using the repetitive cycling method. Significantly, the Co 2p peaks in Fig. 6a indicate that the films produced with this method are not a pure $\mathrm{CoS}_{x}$ film but also contain other oxygenated species like $\mathrm{CoO}$ or $\mathrm{Co}(\mathrm{OH})_{2}$ which appear over the range of 779 to $783 \mathrm{eV} \cdot{ }^{95-101}$ The peak at around $786 \mathrm{eV}$ can be characterized as the shake-up satellite peak of $\mathrm{CoO}$ or $\mathrm{Co}(\mathrm{OH})_{2} \cdot{ }^{102}$ At TU concentrations of 0.5 and $1.0 \mathrm{M}$ another new peak can be observed at $778 \mathrm{eV}$ which has been attributed to the formation of Co-S bonds. ${ }^{76,103}$ Fig. $5 \mathrm{~b}$ represents the $S 2$ p core level spectra where the peaks from 160 to $165 \mathrm{eV}$ can be attributed to the surface absorption of oxygen with sulfur and therefore doping of the $\mathrm{CoS}_{x}$ film with oxygen. ${ }^{76}$

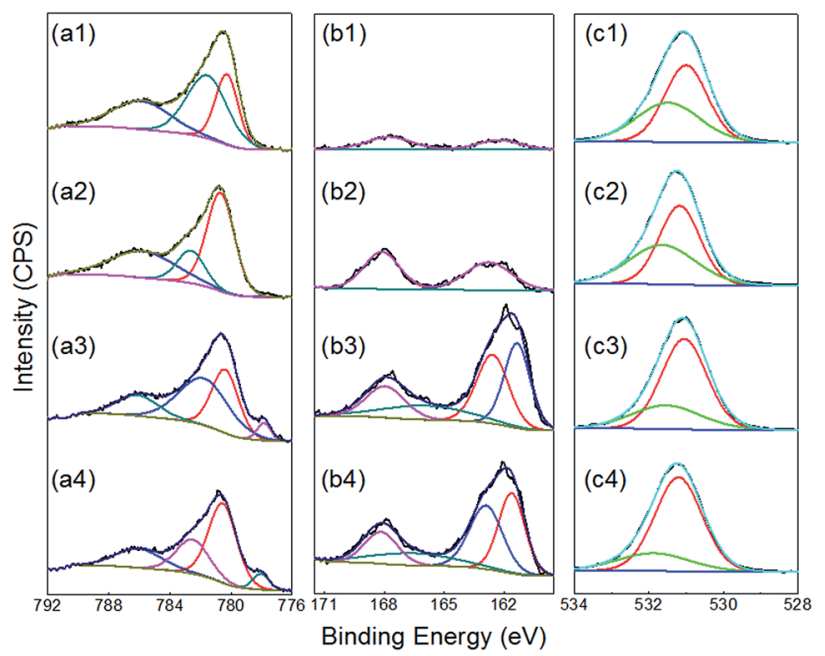

Fig. 6 (a) Co 2p XPS spectra, (b) S 2p XPS, (c) O 1s spectra of deposited films obtained through cyclic voltammetry at a scan rate of $15 \mathrm{mV} \mathrm{s}^{-1}$ for 15 cycles in solutions of $0.005 \mathrm{M} \mathrm{Co(॥)} \mathrm{containing} \mathrm{(a1-c1)} 0.005 \mathrm{M} \mathrm{TU}$, (a2-c2) 0.05 M TU, (a3-c3) 0.5 M TU and (a4-c4) 1.0 M TU at pH 5.6.
It can be seen from the relative intensities of the $S 2 p$ peaks that the intensity of the signal for the Co-S bands increases compared with the surfaced absorbed oxygen with sulfur peak as the TU concentration increases. This is consistent with the Co 2p spectra, which shows a clear increase in the Co-S peak at $778 \mathrm{eV}$. Though the high resolution spectrum of $\mathrm{O} 1 \mathrm{~s}$ bands are often complex and difficult to interpret, ${ }^{104}$ in this study the peaks were fitted as shown in Fig. 6c. The peak at around $531 \mathrm{eV}$ (from the as deposited film) in the spectrum indicates the presence of hydroxides and oxides of $\mathrm{Co},{ }^{104}$ with a slight shift to lower binding energy upon the incorporation of more sulfur in the films.

The composition of all samples was then determined by XPS and is shown in Table 1. A clear trend can be seen in that the concentration of sulfur increases when more TU is added to the electrolyte for both constant potential and repetitive cycling deposition processes, whereby more sulfur is incorporated for the latter approach and is consistent with the electrochemical data. The optimum HER performance was observed for the sample deposited from $5 \mathrm{mM} \mathrm{Co}^{2+}$ with $0.5 \mathrm{M}$ TU using repetitive potential cycling (Fig. 5d). This sample has a Co $: \mathrm{S}$ ratio of 1.56, increasing or decreasing this ratio did not improve performance. Interestingly, when a similar ratio was produced via the chronoamperometric approach the HER performance was quite poor and may be related to the morphology of the film. In Fig. $3 c 2$ it is apparent that this film is very dense compared to the more open and porous structure created using the repetitive cycling protocol (Fig. 4c2) thereby facilitating access to the active sites of the catalyst.

In order to further understand the experimentally-observed highly efficient HER performance of $\mathrm{CoS}_{x}$ compounds, we calculated the hydrogen binding free energy on the $\operatorname{CoS}_{x}$ catalyst as shown in Fig. 7 based on density function theory (DFT). The overall HER can be described as three steps, the initial state $\mathrm{H}^{+}+\mathrm{e}^{-}$, the intermediate adsorbed $\mathrm{H}^{*}$ and the final product $1 / 2 \mathrm{H}_{2} \cdot{ }^{105}$ Hydrogen should not bind catalyst too strong and too weak and the ideal value for $\Delta G_{\mathrm{H}}$ is close to zero. Clearly the value of $\Delta G_{\mathrm{H}}$ for $\operatorname{CoS}_{x}$ was quite negative (Fig. 7), which represent a very strong interaction between adsorbed $\mathrm{H}$ and $\operatorname{CoS}_{x}$. Therefore, it is expected that pristine $\operatorname{CoS}_{x}$ exhibits poor HER reaction kinetics. However, when one $\mathrm{S}$ atom was removed from the Co atom, i.e. the $\mathrm{S}-\mathrm{Co}$ coordination number is reduced, the hydrogen binding

Table 1 Composition ratios for $\mathrm{CoS}_{x}$ electrodeposited from various bath compositions using different electrochemical techniques

\begin{tabular}{|c|c|c|c|c|}
\hline \multirow[b]{2}{*}{ Process } & \multirow[b]{2}{*}{ Bath composition } & \multicolumn{2}{|c|}{ Atomic \% } & \multirow{2}{*}{$\frac{\text { Ratios }}{\mathrm{Co} / \mathrm{S}}$} \\
\hline & & Co & $\mathrm{S}$ & \\
\hline \multirow[t]{4}{*}{$\mathrm{CA}$} & Co $0.005 \mathrm{M}+$ TU $0.005 \mathrm{M}$ & 100 & 0 & - \\
\hline & Co $0.005 \mathrm{M}+$ TU $0.05 \mathrm{M}$ & 99.68 & 0.32 & 311.5 \\
\hline & Co $0.5 \mathrm{M}+\mathrm{TU} 0.5 \mathrm{M}$ & 73.84 & 26.16 & 2.82 \\
\hline & Co $1.0 \mathrm{M}+\mathrm{TU} 1.0 \mathrm{M}$ & 61.42 & 38.58 & 1.59 \\
\hline \multirow[t]{4}{*}{$\mathrm{CV}$} & Co $0.005 \mathrm{M}+\mathrm{TU} 0.005 \mathrm{M}$ & 98.72 & 1.28 & 77.12 \\
\hline & Co $0.005 \mathrm{M}+\mathrm{TU} 0.05 \mathrm{M}$ & 83.04 & 16.96 & 4.89 \\
\hline & Co $0.5 \mathrm{M}+\mathrm{TU} 0.5 \mathrm{M}$ & 60.94 & 39.06 & 1.56 \\
\hline & Co $1.0 \mathrm{M}+\mathrm{TU} 1.0 \mathrm{M}$ & 55.51 & 44.49 & 1.25 \\
\hline
\end{tabular}




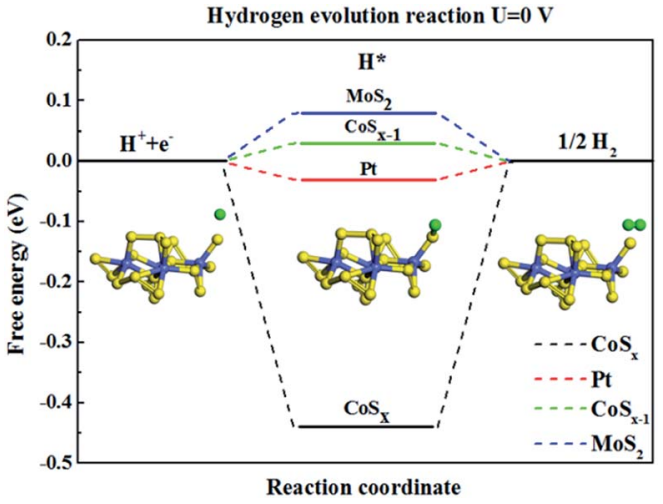

Fig. 7 The calculated free-energy diagram of HER under standard conditions for $\mathrm{CoS}_{x}$ cluster catalysts, and the referenced Pt and $\mathrm{MoS}_{2}$.

free energy $\left(\Delta G_{\mathrm{H}}\right)$ on nearly every $\mathrm{S}$ atom can be reduced to $0.03 \mathrm{eV}$, which is comparable to that of the state-of-the-art $\mathrm{Pt}^{\mathbf{1 0 6}}$ $\left(\Delta G_{\mathrm{H}}=-0.03 \mathrm{eV}\right)$ and $\mathrm{MoS}_{2}$ (ref. 63) $\left(\Delta G_{\mathrm{H}}=0.08 \mathrm{eV}\right)$ catalyst. Our calculations explain the experimentally-observed HER activity when the number of coordinated S-atoms around Co atom is reduced in an amorphous $\operatorname{CoS}_{x}$ compound.

The ability of an electrocatalyst to be functional for both the HER and the OER is highly advantageous and therefore we also tested our material for the OER under alkaline conditions. Fig. 8a shows the OER at each sample produced by cyclic voltammetry at pH 5.6 using different TU concentrations. It illustrates that the samples are quite comparable in the potential $(1.60 \mathrm{~V})$ that is required to generate a current density of $10 \mathrm{~mA} \mathrm{~cm}^{-2}$. For the sample prepared with $0.5 \mathrm{M}$ TU that was active for HER in alkaline solution (Fig. 5d) a Tafel slope of $67 \mathrm{mV} \mathrm{dec}{ }^{-1}$ was determined (Fig. 8b). This is consistent with previous work on oxygen containing $\operatorname{CoS}_{x}$ nanocubes which gave the same Tafel slope in $1.0 \mathrm{M} \mathrm{KOH}$ and achieved the same current density at $1.52 \mathrm{~V}$. The enhanced activity for the OER was predicted by DFT calculations to be due to the presence of oxygen and dangling Co-S bonds. ${ }^{76}$ The main advantage with the approach undertaken here compared to this previous study is that the synthesis can be done rapidly and in one step under ambient conditions where good adherence to the underlying support is achieved. In

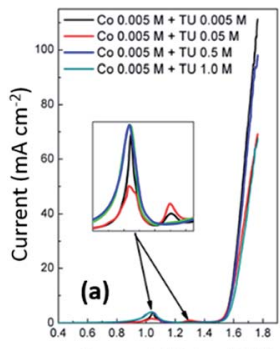

Potential (V vs RHE)

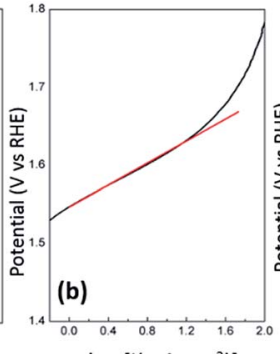

$\log \left[\mathrm{j}\left(\mathrm{mA} \mathrm{cm} \mathrm{cm}^{-2}\right)\right]$

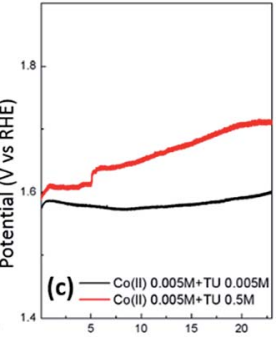

Time (h)
Fig. 8 (a) Linear sweep voltammograms recorded at $5 \mathrm{mV} \mathrm{s}^{-1}$ in $1.0 \mathrm{M}$ $\mathrm{NaOH}$ at samples prepared by cyclic voltammetry at $\mathrm{pH} 5.6$ with different TU concentration, (b) Tafel plot for $\mathrm{CoS}_{x}$ prepared with $0.5 \mathrm{M}$ TU and (c) chronopotentiometric curves recorded at low and high sulfur containing samples where the current density was held at $10 \mathrm{~mA} \mathrm{~cm}^{-2}$ for $24 \mathrm{~h}$. addition electrochemical deposition lends itself to scalability and the ability to be deposited with good adherence on large high surface area electrodes suitable for commercial electrolysis applications. These results also demonstrate that the samples containing higher sulfur content (using $0.5 \mathrm{M}$ TU) perform equally well to the samples with little sulfur content (0.005 M TU) which are essentially cobalt oxide/hydroxide materials which have been documented previously as being active for the OER. ${ }^{107-112}$ Prior to the OER several redox peaks can be seen which are attributed to the oxidation of cobalt into +3 and +4 oxidation states where the latter has been postulated to be the active oxidation state of Co for the OER. ${ }^{108}$ The stability of these catalysts containing low and high $\mathrm{S}$ contents was then tested over a $24 \mathrm{~h}$ period where it can be seen that the sample with a higher sulfur content resulted in a very gradual loss in performance for $18 \mathrm{~h}$ after which it stabilised. This is contrary to the sample with a lower sulfur content which maintained good stability over $24 \mathrm{~h}$.

SEM analysis of the sample prepared with $0.5 \mathrm{M}$ TU after the OER is shown in Fig. $\mathrm{S} 7 \dagger$ which shows two distinct regions which are different to the original starting material (Fig. 4c2). The flake like deposits have been replaced with more nodule like material as well as patches of interconnected plate like materials which are highly indicative of $\mathrm{Co}_{3} \mathrm{O}_{4} \cdot{ }^{113}$ This is supported by XPS analysis (Fig. S8 $\dagger$ ) which shows the emergence of a peak at $529 \mathrm{eV}$ for the $\mathrm{O}$ 1s core level spectrum and suppression of the shake up satellite peak at $788 \mathrm{eV}$ in the $\mathrm{S} 2 \mathrm{p}$ spectrum. The presence of sulfur is still evident, however the intensity of the signal is lower indicating the loss of sulfur from the sample and conversion into a material dominated by surface oxygen as evidenced by the XPS data.

Due to these changes the sample was also investigated after the HER and XPS analysis of the sample after the reaction in alkaline solution shows that the composition of the material is unchanged which is also reflected in the SEM images (Fig. S9†) that indicate no change in the morphology. This is significantly different to the OER under oxidative conditions where there is conversion of the $\operatorname{CoS}_{x}$ material into an oxide material such as $\mathrm{Co}_{3} \mathrm{O}_{4}$ during the course of the reaction which is also an effective catalyst for this reaction. This phenomenon has been highlighted recently by Jin $^{\mathbf{1 1 4}}$ who described that metal chalcogenides, nitrides and phosphides may in fact only be precursors to the active material that participates in the OER. Once these materials are oxidised they are converted into the respective metal oxides/hydroxide which is also evident in this study. Indeed, recent work has utilised metal chalcogenides/ phosphides as a scaffold to produce oxide/hydroxide OER active catalysts on the surface of the original materials which have demonstrated good OER performance. ${ }^{\mathbf{1 1 5 - 1 1 7}}$ Therefore it is important for studies on metal sulphides that the catalyst is characterised post reaction to see if the composition and morphology of the catalyst is maintained rather than changed into a different form that is also active for the reaction of interest.

\section{Conclusions}

The electrodeposition of amorphous $\operatorname{CoS}_{x}$ electrocatalysts containing oxygen could be readily achieved by using a repetitive 
potential cycling method to ensure high sulfur contents once an appropriate $\mathrm{pH}$ of $5-6$ was utilised. The optimum Co : $\mathrm{S}$ ratio found for electrocatalytic activity was $1.56: 1$. This material showed a dual functionality and was active for the HER in acidic, neutral and alkaline media as well as the OER under alkaline conditions once oxidised prior to the reaction which is the key component to ensure good OER performance. DFT calculations confirmed that an amorphous material is appropriate for good HER activity as a reduced S-Co coordination number was found to be better for the optimal adsorption of $\mathrm{H}$. The ease of preparation and activity of this material for both the HER and OER under alkaline conditions is promising for overall electrochemical water splitting.

\section{Conflicts of interest}

There are no conflicts to declare.

\section{Acknowledgements}

The XPS and helium ion microscope data reported in this paper were obtained at the Central Analytical Research Facility operated by the Institute for Future Environments (QUT). Access to CARF is supported by generous funding from the Science and Engineering Faculty (QUT).

\section{Notes and references}

1 J. O. M. Bockris, Science, 1972, 176, 1323.

2 H. Vrubel and X. Hu, ACS Catal., 2013, 3, 2002-2011.

3 P. C. Vesborg, B. Seger and I. Chorkendorff, J. Phys. Chem. Lett., 2015, 6, 951-957.

4 B. J. Fisher and R. Eisenberg, J. Am. Chem. Soc., 1980, 102, 7361-7363.

5 V. Houlding, T. Geiger, U. Kölle and M. Grätzel, Chem. Commun., 1982, 681-683.

6 U. Koelle and S. Paul, Inorg. Chem., 1986, 25, 2689-2694.

7 J. P. Collin, A. Jouaiti and J. P. Sauvage, Inorg. Chem., 1988, 27, 1986-1990.

8 P. V. Bernhardt and L. A. Jones, Inorg. Chem., 1999, 38, 5086-5090.

9 R. Mejia-Rodriguez, D. Chong, J. H. Reibenspies, M. P. Soriaga and M. Y. Darensbourg, J. Am. Chem. Soc., 2004, 126, 12004-12014.

10 A. Begum, G. Moula and S. Sarkar, Chem.-Eur. J., 2010, 16, 12324-12327.

11 Y. Z. Voloshin, A. V. Dolganov, O. A. Varzatskii and Y. N. Bubnov, Chem. Commun., 2011, 47, 7737-7739.

12 W. M. Singh, T. Baine, S. Kudo, S. Tian, X. A. N. Ma, H. Zhou, N. J. DeYonker, T. C. Pham, J. C. Bollinger and D. L. Baker, Angew. Chem., Int. Ed., 2012, 51, 5941-5944.

13 F. Quentel, G. Passard and F. Gloaguen, Energy Environ. Sci., 2012, 5, 7757-7761.

14 D. J. Evans and C. J. Pickett, Chem. Soc. Rev., 2003, 32, 268275.

15 J. W. Tye, M. B. Hall and M. Y. Darensbourg, Proc. Natl. Acad. Sci. U. S. A., 2005, 102, 16911-16912.
16 B. E. Barton, M. T. Olsen and T. B. Rauchfuss, Curr. Opin. Biotechnol., 2010, 21, 292-297.

17 J. L. Dempsey, B. S. Brunschwig, J. R. Winkler and H. B. Gray, Acc. Chem. Res., 2009, 42, 1995-2004.

18 M. Rakowski Dubois and D. L. Dubois, Acc. Chem. Res., 2009, 42, 1974-1982.

19 F. Gloaguen and T. B. Rauchfuss, Chem. Soc. Rev., 2009, 38, 100-108.

20 V. Artero, M. Chavarot-Kerlidou and M. Fontecave, Angew. Chem., Int. Ed., 2011, 50, 7238-7266.

21 M. Wang, L. Chen and L. Sun, Energy Environ. Sci., 2012, 5, 6763-6778.

22 J. L. Inglis, B. J. MacLean, M. T. Pryce and J. G. Vos, Coord. Chem. Rev., 2012, 256, 2571-2600.

23 P. Du and R. Eisenberg, Energy Environ. Sci., 2012, 5, 60126021.

24 A. Le Goff, V. Artero, B. Jousselme, P. D. Tran, N. Guillet, R. Métayé, A. Fihri, S. Palacin and M. Fontecave, Science, 2009, 326, 1384-1387.

25 M. L. Helm, M. P. Stewart, R. M. Bullock, M. R. DuBois and D. L. DuBois, Science, 2011, 333, 863-866.

26 F. Wang, W. G. Wang, X. J. Wang, H. Y. Wang, C. H. Tung and L. Z. Wu, Angew. Chem., Int. Ed., 2011, 50, 3193-3197.

27 Z. Han, F. Qiu, R. Eisenberg, P. L. Holland and T. D. Krauss, Science, 2012, 338, 1321-1324.

28 C. C. McCrory, C. Uyeda and J. C. Peters, J. Am. Chem. Soc., 2012, 134, 3164-3170.

29 E. Anxolabéhère-Mallart, C. Costentin, M. Fournier, S. Nowak, M. Robert and J.-M. Savéant, J. Am. Chem. Soc., 2012, 134, 6104-6107.

30 S. C. Marinescu, J. R. Winkler and H. B. Gray, Proc. Natl. Acad. Sci. U. S. A., 2012, 109, 15127-15131.

31 E. S. Andreiadis, P.-A. Jacques, P. D. Tran, A. Leyris, M. Chavarot-Kerlidou, B. Jousselme, M. Matheron, J. Pécaut, S. Palacin and M. Fontecave, Nat. Chem., 2013, $5,48-53$.

32 Q. Lu, G. S. Hutchings, W. Yu, Y. Zhou, R. V. Forest, R. Tao, J. Rosen, B. T. Yonemoto, Z. Cao and H. Zheng, Nat. Commun., 2015, 6, 6567.

33 X. Wang, R. Su, H. Aslan, J. Kibsgaard, S. Wendt, L. Meng, M. Dong, Y. Huang and F. Besenbacher, Nano Energy, 2015, 12, 9-18.

34 J. R. McKone, B. F. Sadtler, C. A. Werlang, N. S. Lewis and H. B. Gray, ACS Catal., 2013, 3, 166-169.

35 J. Luo, J.-H. Im, M. T. Mayer, M. Schreier, M. K. Nazeeruddin, N.-G. Park, S. D. Tilley, H. J. Fan and M. Grätzel, Science, 2014, 345, 1593-1596.

36 M. Gong, W. Zhou, M.-C. Tsai, J. Zhou, M. Guan, M.-C. Lin, B. Zhang, Y. Hu, D.-Y. Wang and J. Yang, Nat. Commun., 2014, 5, 4695.

37 S. Peng, L. Li, X. Han, W. Sun, M. Srinivasan, S. G. Mhaisalkar, F. Cheng, Q. Yan, J. Chen and S. Ramakrishna, Angew. Chem., 2014, 126, 12802-12807.

38 R. Wu, J. Zhang, Y. Shi, D. Liu and B. Zhang, J. Am. Chem. Soc., 2015, 137, 6983-6986.

39 H. Wang, H.-W. Lee, Y. Deng, Z. Lu, P.-C. Hsu, Y. Liu, D. Lin and Y. Cui, Nat. Commun., 2015, 6, 7261. 
40 M.-R. Gao, Y.-F. Xu, J. Jiang and S.-H. Yu, Chem. Soc. Rev., 2013, 42, 2986-3017.

41 Y. Shi, H. Li and L.-J. Li, Chem. Soc. Rev., 2015, 44, 27442756.

42 H. B. Wu, B. Y. Xia, L. Yu, X.-Y. Yu and X. W. D. Lou, Nat. Commun., 2015, 6, 6512.

43 R. Michalsky, Y.-J. Zhang and A. A. Peterson, ACS Catal., 2014, 4, 1274-1278.

44 Y. Zhao, K. Kamiya, K. Hashimoto and S. Nakanishi, J. Am. Chem. Soc., 2014, 137, 110-113.

45 W. Cui, N. Cheng, Q. Liu, C. Ge, A. M. Asiri and X. Sun, ACS Catal., 2014, 4, 2658-2661.

46 J. Zhu, K. Sakaushi, G. Clavel, M. Shalom, M. Antonietti and T.-P. Fellinger, J. Am. Chem. Soc., 2015, 137, 5480-5485.

47 X. Fan, H. Zhou and X. Guo, ACS Nano, 2015, 9, 5125-5134. 48 W.-F. Chen, C.-H. Wang, K. Sasaki, N. Marinkovic, W. Xu, J. Muckerman, Y. Zhu and R. Adzic, Energy Environ. Sci., 2013, 6, 943-951.

49 E. J. Popczun, C. G. Read, C. W. Roske, N. S. Lewis and R. E. Schaak, Angew. Chem., 2014, 126, 5531-5534.

50 S. Carenco, D. Portehault, C. Boissiere, N. Mezailles and C. Sanchez, Chem. Rev., 2013, 113, 7981-8065.

51 C. G. Morales-Guio, L.-A. Stern and X. Hu, Chem. Soc. Rev., 2014, 43, 6555-6569.

52 Y. Jiao, Y. Zheng, M. Jaroniec and S. Z. Qiao, Chem. Soc. Rev., 2015, 44, 2060-2086.

53 J. Duan, S. Chen, M. Jaroniec and S. Z. Qiao, ACS Nano, 2015, 9, 931-940.

54 Y. Zheng, Y. Jiao, Y. Zhu, L. H. Li, Y. Han, Y. Chen, A. Du, M. Jaroniec and S. Z. Qiao, Nat. Commun., 2014, 5, 3783.

55 C.-Y. Lin, D. Mersch, D. A. Jefferson and E. Reisner, Chem. Sci., 2014, 5, 4906-4913.

56 Y. Sun, C. Liu, D. C. Grauer, J. Yano, J. R. Long, P. Yang and C. J. Chang, J. Am. Chem. Soc., 2013, 135, 17699-17702.

57 N. Kornienko, J. Resasco, N. Becknell, C.-M. Jiang, Y.-S. Liu, K. Nie, X. Sun, J. Guo, S. R. Leone and P. Yang, J. Am. Chem. Soc., 2015, 137, 7448-7455.

58 P. Liu and J. A. Rodriguez, J. Am. Chem. Soc., 2005, 127, 14871-14878.

59 J. Li, X. Zhou, Z. Xia, Z. Zhang, J. Li, Y. Ma and Y. Qu, J. Mater. Chem. A, 2015, 3, 13066-13071.

60 Y. Pan, Y. Lin, Y. Chen, Y. Liu and C. Liu, J. Mater. Chem. A, 2016, 4, 4745-4754.

61 M. Cabán-Acevedo, M. L. Stone, J. Schmidt, J. G. Thomas, Q. Ding, H.-C. Chang, M.-L. Tsai, J.-H. He and S. Jin, Nat. Mater., 2015, 14, 1245-1251.

62 K. Liu, F. Wang, K. Xu, T. A. Shifa, Z. Cheng, X. Zhan and J. He, Nanoscale, 2016, 8, 4699-4704.

63 B. Hinnemann, P. G. Moses, J. Bonde, K. P. Jørgensen, J. H. Nielsen, S. Horch, I. Chorkendorff and J. K. Nørskov, J. Am. Chem. Soc., 2005, 127, 5308-5309.

64 P. Liu, J. Zhu, J. Zhang, P. Xi, K. Tao, D. Gao and D. Xue, ACS Energy Lett., 2017, 2, 745-752.

65 D. Wang, X. Zhang, D. Zhang, Y. Shen and Z. Wu, Appl. Catal., A, 2016, 511, 11-15.

66 T. Liu, Y. Liang, Q. Liu, X. Sun, Y. He and A. M. Asiri, Electrochem. Commun., 2015, 60, 92-96.
67 S. Patra, C. Andriamiadamanana, M. Tulodziecki, C. Davoisne, P.-L. Taberna and F. Sauvage, Sci. Rep., 2016, 6, 21588.

68 P. Montoya, T. Marín, A. Echavarría and J. Calderón, Int. J. Electrochem. Sci., 2013, 8, 12566-12579.

69 A. P. O'Mullane, Nanoscale, 2014, 6, 4012-4026.

70 B. J. Plowman, A. P. O'Mullane and S. K. Bhargava, Faraday Discuss., 2011, 152, 43-62.

71 B. J. Plowman, S. K. Bhargava and A. P. O'Mullane, Analyst, 2011, 136, 5107-5119.

72 I.-W. Sun and J.-K. Chang, in Springer Handbook of Electrochemical Energy, ed. C. Breitkopf and K. SwiderLyons, Springer Berlin Heidelberg, Berlin, Heidelberg, 2017, DOI: 10.1007/978-3-662-46657-5_26, pp. 835-895.

73 T. M. Braun and D. T. Schwartz, Electrochem. Soc. Interface, 2016, 25, 69-73.

74 P. Ganesan, A. Sivanantham and S. Shanmugam, J. Mater. Chem. A, 2016, 4, 16394-16402.

75 A. Sivanantham, P. Ganesan and S. Shanmugam, Adv. Funct. Mater., 2016, 26, 4661-4672.

76 P. Cai, J. Huang, J. Chen and Z. Wen, Angew. Chem., Int. Ed., 2017, 56, 4858-4861.

77 G. Kresse and J. Furthmüller, Comput. Mater. Sci., 1996, 6, 15-50.

78 P. Vogt, P. De Padova, C. Quaresima, J. Avila, E. Frantzeskakis, M. C. Asensio, A. Resta, B. Ealet and G. Le Lay, Phys. Rev. Lett., 2012, 108, 155501.

79 J. P. Perdew, K. Burke and M. Ernzerhof, Phys. Rev. Lett., 1996, 77, 3865.

80 J. P. Perdew, M. Ernzerhof and K. Burke, J. Chem. Phys., 1996, 105, 9982-9985.

81 P. E. Blöchl, Phys. Rev. B, 1994, 50, 17953.

82 S. Grimme, J. Comput. Chem., 2006, 27, 1787-1799.

83 C. Tsai, F. Abild-Pedersen and J. K. Nørskov, Nano Lett., 2014, 14, 1381-1387.

84 A. A. Smirnova, Zool. Zh., 2011, 90, 803-810.

85 A. R. Harris, A. K. Neufeld, A. P. O'Mullane, A. M. Bond and R. J. S. Morrison, J. Electrochem. Soc., 2005, 152, C577.

86 J. Santos, R. Matos, F. Trivinho-Strixino and E. Pereira, Electrochim. Acta, 2007, 53, 644-649.

87 S. Jiang, Y. Chen, J. You, T. Chen and A. Tseung, J. Electrochem. Soc., 1990, 137, 3374-3380.

88 A. Irshad and N. Munichandraiah, ACS Appl. Mater. Interfaces, 2017, 9, 19746-19755.

89 J. A. García-Valenzuela, Comments Inorg. Chem., 2017, 37, 99-115.

90 M. Nicol and V. Tjandrawan, Hydrometallurgy, 2014, 150, 34-40. 91 J. Shi, X. Li, G. He, L. Zhang and M. Li, J. Mater. Chem. A, 2015, 3, 20619-20626.

92 C. K. Ranaweera, Z. Wang, E. Alqurashi, P. K. Kahol, P. R. Dvornic, B. K. Gupta, K. Ramasamy, A. D. Mohite, G. Gupta and R. K. Gupta, J. Mater. Chem. A, 2016, 4, 9014-9018. 93 X. Zou and Y. Zhang, Chem. Soc. Rev., 2015, 44, 5148-5180. 94 H. Zhang, Y. Li, G. Zhang, P. Wan, T. Xu, X. Wu and X. Sun, Electrochim. Acta, 2014, 148, 170-174.

$95 \mathrm{~J}$. Chastain, R. C. King and J. Moulder, Handbook of X-ray photoelectron spectroscopy: a reference book of standard spectra for identification and interpretation of XPS data, Physical Electronics, Eden Prairie, MN, 1995. 
96 N. McIntyre and M. Cook, Anal. Chem., 1975, 47, 2208-2213. 97 T. Jit, K. Beng, J. Kenneth and P. Sherwood, J. Am. Chem. Soc., 1991, 113, 855.

98 M. Wang, A. M. Anghel, B. Marsan, N.-L. Cevey Ha, N. Pootrakulchote, S. M. Zakeeruddin and M. Grätzel, J. Am. Chem. Soc., 2009, 131, 15976-15977.

99 J.-Y. Lin, J.-H. Liao and S.-W. Chou, Electrochim. Acta, 2011, 56, 8818-8826.

100 B. J. Tan, K. J. Klabunde and P. M. Sherwood, J. Am. Chem. Soc., 1991, 113, 855-861.

101 A. M. de Jong, V. De Beer, J. R. van Veen and J. Niemantsverdriet, J. Phys. Chem., 1996, 100, 17722-17724.

102 K. Kim, Phys. Rev. B, 1975, 11, 2177.

103 H. Li, Y. Gao, Y. Shao, Y. Su and X. Wang, Nano Lett., 2015, 15, 6689-6695.

104 J. Yang, H. Liu, W. N. Martens and R. L. Frost, J. Phys. Chem. C, 2009, 114, 111-119.

105 J. K. Nørskov, T. Bligaard, J. Rossmeisl and C. H. Christensen, Nat. Chem., 2009, 1, 37-46.

106 J. K. Nørskov, T. Bligaard, A. Logadottir, J. Kitchin, J. G. Chen, S. Pandelov and U. Stimming, J. Electrochem. Soc., 2005, 152, J23-J26.
107 B. H. R. Suryanto, X. Lu, H. M. Chan and C. Zhao, RSC Adv., 2013, 3, 20936-20942.

108 B. S. Yeo and A. T. Bell, J. Am. Chem. Soc., 2011, 133, 55875593.

109 M. E. G. Lyons and M. P. Brandon, J. Electroanal. Chem., 2010, 641, 119-130.

110 M. A. Sayeed, T. Herd and A. P. O'Mullane, J. Mater. Chem. A, 2016, 4, 991-999.

111 X. Zhou, Z. Xia, Z. Tian, Y. Ma and Y. Qu, J. Mater. Chem. A, 2015, 3, 8107-8114.

112 Y. Zhao, S. Chen, B. Sun, D. Su, X. Huang, H. Liu, Y. Yan, K. Sun and G. Wang, Sci. Rep., 2015, 5, 7629.

113 N. Zhang, Y. Fan, H. Fan, H. Shao, J. Wang, J. Zhang and C. Cao, ECS Electrochem. Lett., 2012, 1, H8-H10.

114 S. Jin, ACS Energy Lett., 2017, 2, 1937-1938.

115 X. Xu, F. Song and X. Hu, Nat. Commun., 2016, 7, 12324.

116 W. Chen, H. Wang, Y. Li, Y. Liu, J. Sun, S. Lee, J.-S. Lee and Y. Cui, ACS Cent. Sci., 2015, 1, 244-251.

117 H. Liang, A. N. Gandi, C. Xia, M. N. Hedhili, D. H. Anjum, U. Schwingenschlögl and H. N. Alshareef, ACS Energy Lett., 2017, 2, 1035-1042. 